\title{
Special Issue on Heterocyclic Chemistry
}

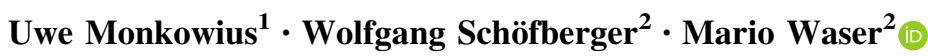

Received: 14 January 2018/ Accepted: 16 January 2018/Published online: 23 March 2018

(c) Springer-Verlag GmbH Austria, part of Springer Nature 2018

Dear readers,

This special issue of Chemical Monthly is dedicated to heterocyclic chemistry in all its variations. As (organic) chemists we are all aware of the uttermost importance of heterocyclic compounds in our daily life and well-being. It is thus no surprise that numerous research groups focus on investigations concerning syntheses and applications of heterocycles, resulting in spectacular achievements and developments in this field. From August 30 until September 2, 2017 the three of us had the honor to organize the 17th Blue Danube Symposium on Heterocyclic Chemistry at the Johannes Kepler University Linz, Austria. These Blue Danube symposia have become a well-established medium-sized international meeting series and its interesting history, i.e. the ideas and visions of scientists from Austria, Czech Republic, Hungary, Slovakia and Slovenia to establish these symposia, are very nicely outlined in the following editorial of this issue by Marko Mihovilovic. In the course of this symposium, which was attended by more than 160 participants from 25 countries and 5 continents, we had the chance to witness the strength and beauty of heterocyclic chemistry in all its variations. Inspired by the important achievements presented in this conference, we decided to set up this special issue in which we have tried to bring together articles covering the various aspects of heterocyclic chemistry. May it be more target interested research like, e.g. natural product synthesis and drug

Mario Waser

mario.waser@jku.at

1 School of Education, Johannes Kepler University Linz, Altenbergerstraße 69, 4040 Linz, Austria

2 Institute of Organic Chemistry, Johannes Kepler University Linz, Altenbergerstraße 69, 4040 Linz, Austria development, or more method development focused articles on, e.g. (asymmetric) catalysis, we are convinced that within this issue you will find articles giving you an interesting overview about the latest achievements in the fascinating field of heterocyclic chemistry. All these contributions were written by authors on personal invitation, with some, but not all of them, being participants at the Blue Danube Symposium. We are really grateful that so many followed our invitations and provided such an illustrative overview of the field, not only by contributing recent research articles, but also by taking the efforts in contributing some review articles on different topics in heterocyclic chemistry. We are also grateful to the authors and reviewers for submitting their articles/reports very well in time, which made the handling for us a real pleasure. We also wish to thank Peter Gärtner and Christian Hametner for their support during the preparation of this special issue.

Very sadly however, we cannot close our editorial at this point, because just during the finalization of this article we became aware of the sad and shocking news that Prof. Walther Schmid (University of Vienna) passed away far too early on December 28, 2017. Prof. Schmid was a wonderful and inspiring teacher, mentor, colleague, and friend of many of the authors that contributed to this special issue and on behalf of so many who had the honor and privilege of knowing him we wish to dedicate this special issue to his memory.

Sincerely,

Uwe Monkowius, Wolfgang Schöfberger, Mario Waser 